Tsunenori Hirayama • Emi Yamaki • Akira Hata Masahiro Tsuji - Kiyoshi Hashimoto - Masao Yamamoto Mitsuru Emi

\title{
Five familial hypercholesterolemic kindreds in Japan with novel mutations of the LDL receptor gene
}

\begin{abstract}
In the course of investigations of familial coronary artery disease in Hokkaido, the northern island of Japan, we identified five families in which multiple members showed elevated plasma levels of low-density lipoprotein (LDL) cholesterol. To determine the genetic etiology of their lipoprotein abnormalities, we screened DNA samples from these families for mutations in all 18 exons and the exon-intron boundaries of the $L D L$ receptor $(L D L R)$ gene. Novel point mutations were identified in each family: (1) a C-to-A transversion at nucleotide 285 , causing a nonsense mutation at codon 74 , in eight members of family A; (2) a G-to-A transition at nucleotide 1136, causing substitution of Tyr for Cys at codon 358, in six members of family B; (3) a C-to-T transition at nucleotide 1822, causing substitution of Ser for Pro at codon 587, in five members of family C; (4) a one-base insertion of $\mathrm{G}$ to a fiveG stretch at nucleotides 1774-1778 (codons 571-572), causing a frameshift, in six members of family $\mathrm{D}$; and (5) a one-base deletion of $\mathrm{T}$ at nucleotide 1963-1964 (codon 634), causing a frameshift, in three members of family E. Through the molecular genetic approach a total of 28 individuals in these families were diagnosed unequivocally as heterozygous for the respective LDLR mutations. This method also helped us to diagnose familial hypercholesterolemia, or to exclude from carrier status, 11 children with borderline high cholesterol levels.
\end{abstract}

Key words Hyperlipoproteinemia $\cdot$ Lipoproteins $\cdot$ LDL receptor $\cdot$ Familial hypercholesterolemia $\cdot$ Genetic diagnosis

T. Hirayama $\cdot$ E. Yamamki $\cdot$ M. Emi $(\bowtie)$

Department of Molecular Biology, Institute of Gerontology and

T. Hirayama $\cdot$ K. Hashimoto $\cdot$ M. Yamamoto

Department of Pediatrics, Nippon Medical School, 1-396, Kosugi-

cho, Nakahara-ku, Kawasaki-shi, Kanagawa 211-8533, Japan.

Tel. +81-44-733-5230; Fax +81-44-733-5192

e-mail:memi@nms.ac.jp

A. Hata

Department of Public Health, Hokkaido University Sapporo, Japan

M. Tsuji

Hokkaido Central Hospital for Social Health Insurance, Sapporo, Japan

\section{Introduction}

Plasma lipid and lipoprotein levels reflect in part the influence of relevant genetic loci. Defects at such loci account for specific types of dyslipoproteinemia that may occur with regularity among members of some families. Heterozygous familial hypercholesterolemia $(\mathrm{FH})$ results from the inheritance of a single defective copy of a low-density lipoprotein receptor (LDLR) gene (Goldstein and Brown, 1989). The disease is recognized clinically by striking elevations of LDL cholesterol (about twice the normal range, or $>95$ th percentile) in children as well as adults. Affected families display bimodally distributed LDL cholesterol levels consistent with an autosomal dominant trait. Typically, there is a striking family history of early coronary disease. In general, only LDL cholesterol levels are elevated in FH; elevated triglycerides or other lipid abnormalities may bring into question the diagnosis of FH and may suggest other inherited primary lipid disorders such as familial combined hyperlipidemia, or secondary lipid disorders.

The $L D L R$ gene is large and structually complex; it spans $45 \mathrm{~kb}$ of the human genome and consists of 18 exons and 17 introns (Sudhof et al. 1985). No mutational "hotspot" has been identified; in fact, specific mutations rarely recur, either among unrelated Caucasians or among families residing in mainland Japan (Hobbs et al. 1990, 1992). The LDLR mutations have been classified into the following five classes on the basis of biosynthetic and functional studies of fibroblasts derived from FH patients: null alleles (class 1 mutations), transport-defective alleles (class $2 \mathrm{mu}-$ tations), binding-defective alleles (class 3 mutations), internalization-defective alleles (class 4 mutations), and recycling-deficient alleles (class 5 mutations) (Russell et al. 1989; Hobbs et al. 1990, 1992).

We describe here the identification of novel mutations of the $L D L R$ gene in each of five hypercholesterolemic kindreds in the north of Japan. Our molecular genetic approach allowed as to diagnose 28 individuals in these families as having $\mathrm{FH}$ and also aided in the diagnosis of children with borderline high cholesterol levels. 


\section{Patients and methods}

Patients and lipoprotein measurement

Five families (A through E) that were anecdotally known to have members with hyperlipidemia were referred to one of us (M. T.) and followed clinically at the outpatient clinic of Hokkaido Central Hospital for Social Health Insurance. Blood samples collected after $12-16 \mathrm{~h}$ of fasting were prepared from each family member. Lipid and lipoprotein concentrations were measured by procedures described previously (Williams et al. 1986). In brief, plasma cholesterol and triglyceride concentrations were assayed enzymatically, and concentrations of high-density lipoprotein (HDL) cholesterol were determined by the $\mathrm{MgCl}_{2}$-dextran precipitation method.

Polymerase chain reaction (PCR)-single-strand conformation polymorphism (SSCP) and sequencing analyses

Each PCR was carried out using 20ng of genomic DNA extracted from lymphocytes, in a $10-\mu$ l aliquot of solution containing 10mM Tris- $\mathrm{HCl}(\mathrm{pH} 8.4), 50 \mathrm{mM} \mathrm{KCl}, 1.5 \mathrm{mM}$ $\mathrm{MgCl}_{2}, 0.01 \%$ gelatin, $200 \mu \mathrm{M}$ each of deoxyribonucleotide triphasphate (dNTP), $2 \mu \mathrm{Ci}$ of [alpha 32P] deoxycytidinetriphosphate (dCTP) $(3000 \mathrm{Ci} / \mathrm{mmol}, 10 \mathrm{mCi} / \mathrm{ml}), 2.5 \mathrm{pmol}$ of each primer, and 0.25 units of Taq polymerase. PCR primers for amplification of each exon of the $L D L R$ gene and all exon-intron boundaries were described previously by Leitersdorf et al. (1990) and Leren et al. (1993). Each of $35 \mathrm{PCR}$ cycles consisted of $30 \mathrm{~s}$ at $94^{\circ} \mathrm{C}, 30 \mathrm{~s}$ at $60^{\circ} \mathrm{C}$, and $30 \mathrm{~s}$ at $72^{\circ} \mathrm{C}$. Reaction mixtures were diluted with $50 \mu \mathrm{l}$ of $95 \%$ formamide dye and $20 \mathrm{mM}$ ethylene diametric tetraacetate (EDTA), incubated at $85^{\circ} \mathrm{C}$ for $5 \mathrm{~min}$, and applied $(2 \mu \mathrm{l}$ per lane) to $6 \%$ polyacrylamide gels containing $0.5 \times \mathrm{TBE}$ (90 mM Tris-borate $/ 2 \mathrm{mM}$ EDTA) and 5\% glycerol. Electrophoresis was performed both at room temperature and at $4^{\circ} \mathrm{C}$. The gels were dried and autoradiographed with intensifying screens. When a variant band was revealed by SSCP analysis, the corresponding DNA fragment was sequenced directly on both strands to identify the nature of the mutation. The results were confirmed by two independent experiments.

\section{Results}

Family A: C74X in exon 3

The pedigree structure of family $\mathrm{A}$ is shown in Fig. 1, along with their sex, age, lipoprotein profile, and affected status as determined by the mutation analysis. Screening of the $L D L R$ gene in the proband's genomic DNA identified an aberrant SSCP pattern in exon 3; direct sequencing revealed heterozygosity for a C-to-A transversion at nucleotide position 285 (Fig. 1a). This transversion would cause a
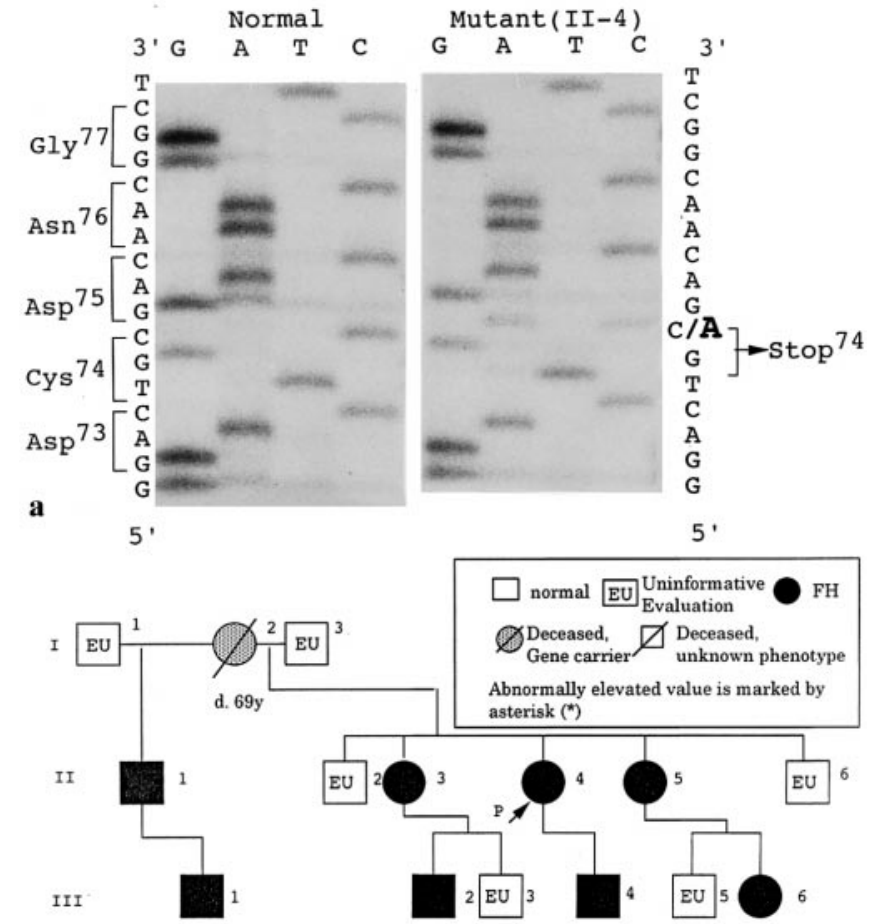

b I-1 II-1 III-1 I-2 I-3 II-2 II-3III-2 III-3 II-4 III-4 II-5 III-5 III-6 II-6

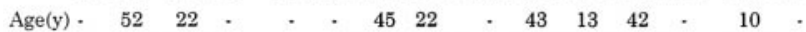
TC $-7.73^{*} 6.98^{*}$. $\quad 7.11^{*} 7.24^{*} \cdot 9.72^{*} 8.09^{*} 8.09^{*} \cdot 7.93^{*}$. TG $\quad 0.351 .22 \cdot \quad \cdot \quad \cdot 2.77^{\star} \cdot \quad \cdot 2.19^{*} 0.960 .66 \quad-1.47$ LDLC - $3.74^{\star} 5.42^{\star}$. $\quad .4 .91^{*}$. $4.88^{\star} 5.94^{\star} 5.94^{\star} \cdot 4.73^{*}$ HDLC - $3.82^{*} 0.98$ - $\quad-0.93$ - 0.961 .451 .34 - 0.16 .

Fig. 1 a Mutational analysis and b pedigree of the proband ( $p$, arrow) in family A. Normal ranges of plasma lipoprotein levels for the Japanese population are: Total cholesterol $(T C),<5.68 \mathrm{mmol} / \mathrm{l}$; triglyceride $(T G),<1.69 \mathrm{mmol} / \mathrm{l}$; low-density lipoprotein cholesterol (LDLC), $<3.62 \mathrm{mmol} / \mathrm{l}$; high-dersity lipoprotein cholesterol (HDLC), $<1.55 \mathrm{mmol} / 1$. Square symbols, Males; circles, females; $F H$, familial hypercholesterolemia

nonsense mutation, replacing a cysteine at codon 74 , and would yield a truncated protein. The mutation was found in eight members of the family, including the proband.

The mean levels of total cholesterol, total triglyceride, LDL cholesterol, and HDL cholesterol among these eight carriers were $8.24,1.65,6.07$, and $1.16 \mathrm{mmol} / 1$, respectively. Two children suspected to have the disease, a 13-year-old boy (III-4) and a 10-year-old girl (III-6), were indeed found to carry the mutation and were thus unequivocally diagnosed with FH.

\section{Family B: C358Y in exon 8}

The pedigree structure of family $B$ is shown in Fig. 2. An aberrant SSCP pattern was identified in exon 8 of the LDLR in the proband's genomic DNA, and direct sequencing revealed heterozygosity for a G-to-A transition at nucleotide 1136 (Fig. 2a). This transition would substitute tyrosine for cysteine at codon 358, abolishing a critical cysteine residue in the first Y-repeat of the EGF-precursor homology domain. The mutation was found in six members of the family, including the proband. 

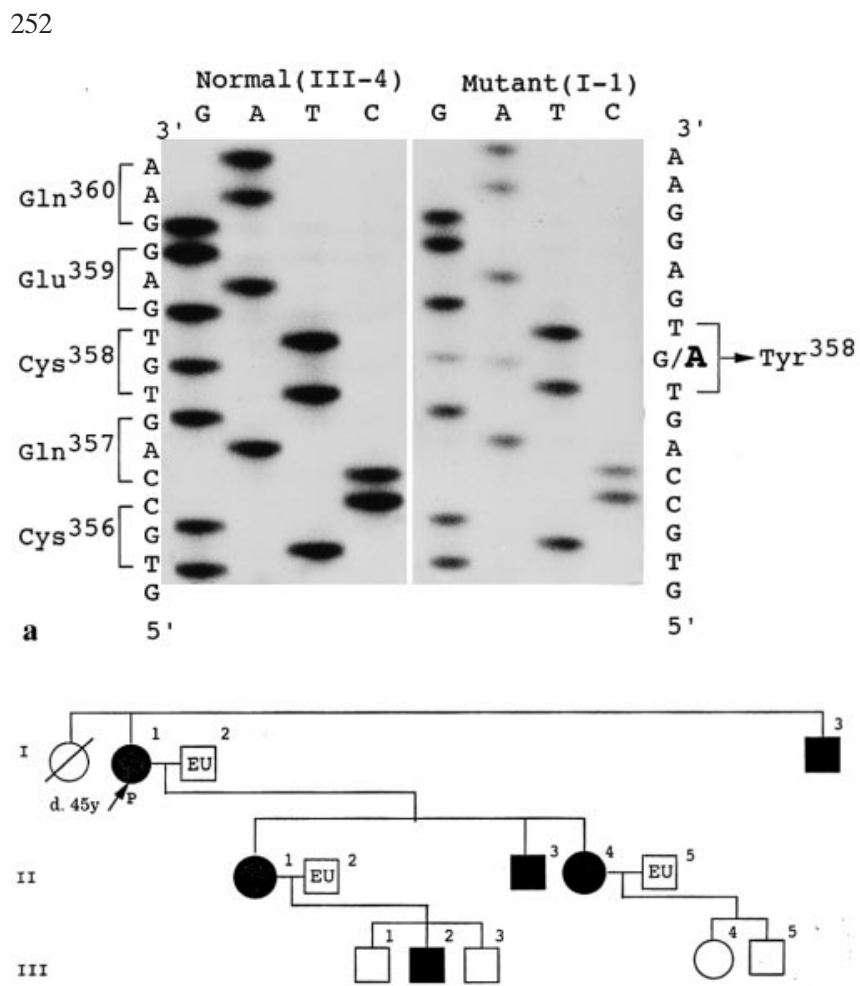
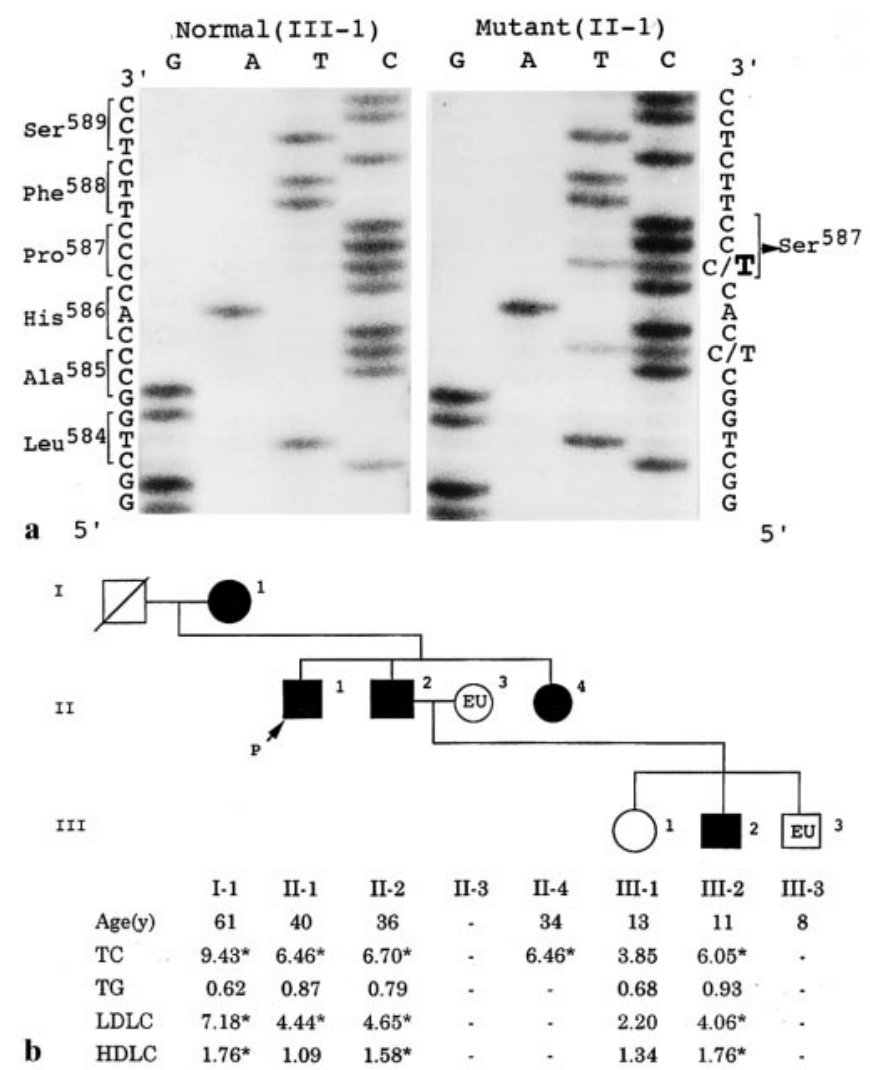

Fig. 3 a Mutational analysis and $\mathbf{b}$ pedigree of the proband ( $p$, arrow) in family C. Keys to symbols are as in Fig. 1 and its legend. Normal ranges of plasma lipoprotein levels are as described in Fig. 1 legend

Fig. 2 a Mutational analysis and $\mathbf{b}$ pedigree of the proband ( $p$, arrow) in family B. Keys to symbols are as in Fig. 1 and its legend. Normal ranges of plasma lipoprotein levels, are as described in Fig. 1 legend

The mean levels of total cholesterol, total triglyceride, LDL cholesterol, and HDL cholesterol among these six carriers were $8.17,1.64,6.23$, and $1.09 \mathrm{mmol} / 1$, respectively. Three children in this family showed equivocal levels of plasma cholesterol (i.e., 7.36, 5.45, 4.08 $\mathrm{mmol} / \mathrm{l}$ ); one of them, a 4-year-old boy (III-2), did carry the mutation but the others, a 4-year-old girl (III-4) and a 1-year-old boy (III-5), did not.

\section{Family C: P587S in exon 12}

The pedigree structure of family $\mathrm{C}$ is shown in Fig. 3. The proband was heterozygous for a C-to-T transition at nucleotide 1822 (Fig. 3a), which would result in substitution of serine for proline at codon 587 in the fourth Y-repeat of the EGF precursor homology domain. The mutation was found in five members of the proband's family.

The mean levels of total cholesterol, total triglyceride, LDL cholesterol, and HDL cholesterol among these five carriers were $7.16,0.80,5.09$, and $1.55 \mathrm{mmol} / 1$, respectively. A 11-year-old boy (III-2), who was suspected to have the disease in view of a plasma cholesterol level of $6.05 \mathrm{mmol} / 1$, was found to carry the mutation. A rare polymorphism, a Cto-T transition at the third nucleotide of 585Ala, was ob-

served as well. This silent variant was carried on the same allele as the P587S mutation, and co-segregated with it in family C.

\section{Family D: 1773 insG in exon 12}

The pedigree structure of family $\mathrm{D}$ is shown in Fig. 4. The proband was heterozygous for a one-base $(\mathrm{G})$ insertion in a stretch of five guanines at nucleotides 1774-1778 (codons 571/572) (Fig. 4a). This insertion would cause a frameshift resulting in premature termination $30 \mathrm{bp}$ downstream, at codon 581. The mutation was found in six members of the family, including the proband.

The mean levels of total cholesterol, total triglyceride, LDL cholesterol, and HDL cholesterol among these six carriers were $8.09,1.55,5.89$, and $1.21 \mathrm{mmol} / 1$, respectively. Of the four children in this family, one boy at age 16 (III-3) was suspected to have the disease, having a total cholesterol level of $6.41 \mathrm{mmol} / \mathrm{l}$, but definitive diagnosis was difficult on purely clinical grounds. The present study showed that the child does carry the mutation and therefore can be diagnosed unambiguously with FH. Of the three remaining children, an 18-year-old boy (III-2) and a 10-year-old boy (III-4) were diagnosed as affected and a 19-year-old girl (III-1) was diagnosed as normal, consistent with their clinical diagnoses. 

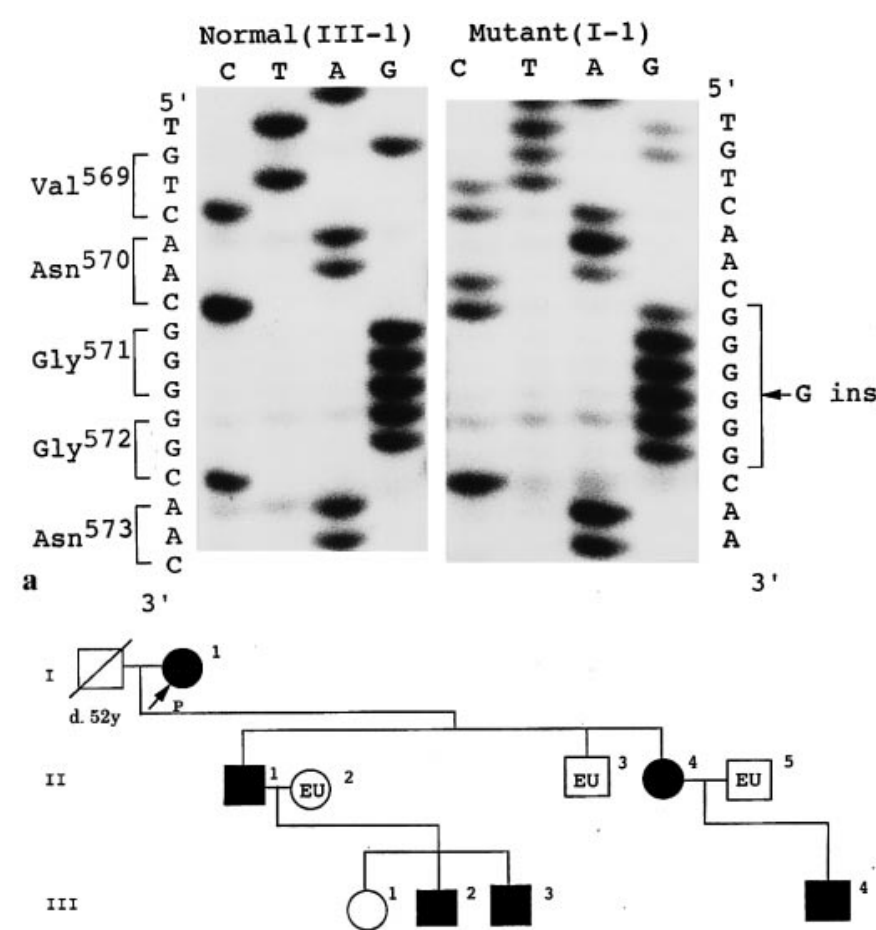

\begin{tabular}{|c|c|c|c|c|c|c|c|c|c|c|}
\hline & I-1 & II-1 & II-2 & III-1 & III-2 & III-3 & II-3 & II-4 & II-5 & III-4 \\
\hline $\mathrm{Age}(\mathrm{y})$ & 66 & 45 & 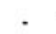 & 19 & 18 & 16 & 42 & 31 & - & 10 \\
\hline $\mathrm{TC}$ & $7.11^{\star}$ & $8.39^{\star}$ & . & 3.75 & $8.01^{*}$ & $6.41^{*}$ & . & $8.50^{\star}$ & . & $9.90^{*}$ \\
\hline $\mathrm{TG}$ & $2.46^{*}$ & $2.28^{*}$ & . & 0.69 & 0.46 & 0.53 & . & 1.56 & $\therefore$ & $1.97^{\star}$ \\
\hline LDLC & $5.06^{*}$ & $6.12^{*}$ & . & 2.27 & $6.07^{\star}$ & $4.39^{\star}$ & . & $6.56^{\star}$ & . & $7.13^{*}$ \\
\hline HDLC & 0.78 & 0.85 & . & 1.16 & 1.27 & 1.27 & . & 1.21 & . & $1.86^{*}$ \\
\hline
\end{tabular}

Fig. 4 a Mutational analysis and b pedigree of the proband ( $p$, arrow) in family D. Keys to symbols are as in Fig. 1 and its legend. Normal ranges of plasma lipoprotein levels are as described in Fig. 1 legend

Family E: 1963delT in exon 13

The pedigree structure of family $\mathrm{E}$ is shown in Fig. 5. Screening of exon 13 of $L D L R$ revealed that the proband was heterozygous for a one-base deletion $(\mathrm{T})$ at nucleotide position 1963 in codon 634 (Fig. 5a). This deletion would cause a frameshift resulting in premature termination at codon 643 . The mutation was found in three members of the family, including the proband.

The mean levels of total cholesterol, total triglyceride, LDL cholesterol, and HDL cholesterol among these three carriers were $7.75,0.85,6.23$, and $0.93 \mathrm{mmol} / 1$, respectively. The 7-year-old son (III-1) of an FH-affected father was diagnosed as normal both by lipid profile and molecular analysis. A T-to-C polymorphism at the third nucleotide of $632 \mathrm{Val}$ was observed in the proband of family E. This silent variant did not co-segregate with the 1963delT mutation.

\section{Discussion}

Environmental as well as genetic factors influence lipid levels in plasma. Japanese people in general have lower cholesterol levels than Western populations, owing to their
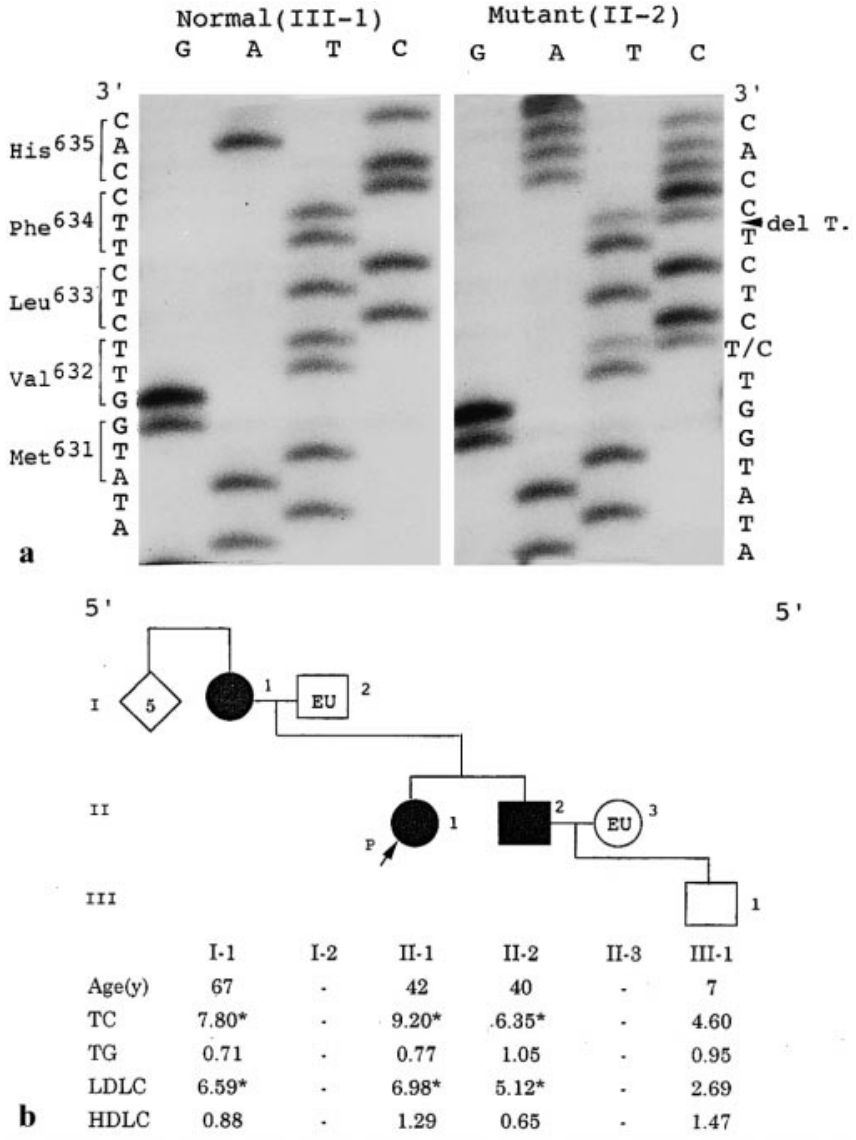

Fig. 5 a Mutational analysis and $\mathbf{b}$ pedigree of the proband ( $p$, arrow) in family E. Keys to symbols are as in Fig. 1 and its legend. Normal ranges of plasma lipoprotein levels are as described in Fig. 1 legend

traditionally low-fat, high-carbohydrate diets (Mabuchi et al. 1977). This may explain the comparatively lower levels of LDL cholesterol seen in Japanese FH patients (Maruyama et al. 1995). The nature of the LDL receptor mutation also contributes to clinical variability among FH patients: individuals with mutations that only partially impair receptor function tend to have lower plasma LDL cholesterol levels than do individuals whose genes produce totally defective receptors (Goldstein and Brown 1989).

In the present study, $\mathrm{FH}$ patients with the missense mutation S587P in family $\mathrm{C}$ tended to have levels of total cholesterol and LDL cholesterol 15\%-20\% lower than levels in $\mathrm{FH}$ patients of similar age and sex whose mutations caused protein truncations; i.e., the nonsense or frameshift mutations seen in families A (C74X), D (1773insG), and E (1963delT). FH patients in family B, who carry a different missense mutation (C358Y), had comparatively higher cholesterol levels. In this connection it is worth noting that replacement of the critical cysteine residue at codon 358 by another amino acid residue, arginine, completely abolished receptor recycling in a previously reported $\mathrm{FH}$ patient, as described below (FH Naples-1, C358A; Hobbs et al. 1992).

Functionally, on the basis of studies that involved fibroblasts derived from FH patients (Russell et al. 1989; Hobbs et al. 1990, 1992), LDLR mutations were classified into five 
classes. Class 1 alleles fail to produce immunoprecipitable LDL receptor protein and are called null alleles. In the present study, we detected three nonsense or frameshift mutants; C74X in family A, 1773insG in family D, and 1963delT in family E. Since these alterations would fail to produce intact receptor, they are class 1 mutations (null alleles). Nonsense and frameshift mutations are the most frequent types of class 1 alterations reported, and they tend to be randomly distributed among the exons of LDLR. Class 2 alleles encode proteins that are blocked either completely or partially in transport between the endoplasmic reticulum and the Golgi apparatus. We observed a substitution of serine for proline at codon 587 in the fourth Y-repeat of the EGF precursor homology domain in family C. Four other missense mutants occurring in the fourth Y-repeat of the EGF- precursor homology domain encoded by exon 12 have previously been functionally characterized (Hobbs et al. 1992). Since most of them encoded receptors that were blocked in transport between the endoplasmic reticulum and the Golgi apparatus (class 2; transport-defective alleles), we postulate that the P587S mutation reported here would cause the same class of deficiency, because of its location. Other possibilities cannot be ruled out, however, until proper functional tests are conducted for this particular mutation.

Class 5 alleles encode receptors that do not recycle to the cell surface because they fail to release the ligands in the endosome. In family $\mathrm{B}$, we observed a substitution of tyrosine for cysteine at codon 358, abolishing a critical cysteine residue in the first Y-repeat of the EGF-precursor homology domain. The $5^{\prime}$ end of the EGF-precursor homology domain, especially in the first Y1-repeat region, mediates acid-dependent dissociation of receptor and ligand in the endosome, an event essential for receptor recycling (Davis et al. 1987). The cysteine residue at codon 358 appears to be critical for receptor recycling, in view of the report by Hobbs et al. (1992) that LDL receptors on fibroblasts from patient FH Naples-1, who carried a Cys- toArg substitution at the same residue, failed to release the ligands in the endosome and thus did not recycle to the cell surface. In fact, class 5 mutations (recycling-deficient alleles) cluster characteristically in the Y1-repeat region of the EGF-precursor homology domain (Hobbs et al. 1992). In addition to the three classes of mutants observed in the present study, two other types of mutation have been characterized; class 3 alleles that encode proteins that fail to bind LDL normally, and class 4 alleles that encode receptors that are unable to cluster in clathrin-coated pits and thus do not internalize LDL (Hobbs et al. 1992).

Hypercholesterolemia is sometimes not fully expressed in the children of FH families until they are older. Since such children may show borderline high levels with respect to their age groups, definitive diagnosis can be difficult on purely clinical grounds, and they may not receive the necessary dietary or medical interventions. In the study reported here, we were able to provide unequivocal diagnosis of children belonging to $\mathrm{FH}$ families, by means of mutational analysis. Our study illustrates the feasibility of molecular genetic approaches in strengthening clinical diagnosis (Nakamura 1996, Yanase 1997) and invoking appropriate management of young members of families with $\mathrm{FH}$, with a view toward early intervention in individuals with a predisposition to coronary heart disease.

Acknowledgments This study was supported by a Grant-in-Aid from the Ministry of Health and Welfare of Japan; and a Grant-in-Aid from the Ministry of Education, Science, Sports, and Culture of Japan.

\section{References}

Davis CG, Goldstein JL, Sudhof TC, Andrson RGW, Russell DW, Brown MS (1987) Acid-dependent ligand dissociation and recycling of LDL receptor mediated by growth factor homology region. Nature 326: 760-765

Goldstein JL, Brown MS (1989) Familial hypercholesterolemia. In: Scriver CR, Beaudet AL, Sly DS, Valle D (eds) The metabolic basis of inherited disease. McGraw-Hill, New York, pp 1215-1250

Hobbs HH, Brown MS, Goldstein JL (1992) Molecular genetics of the LDL receptor gene in familial hypercholesterolemia. Hum Mutation 1: 445-466

Hobbs HH, Russell DW, Brown MS, Goldstein JL (1990) The LDL receptor locus in familial hypercholesterolemia: Mutational analysis of a membrane protein. Annu Rev Genet 24: 133-170

Leitersdorf E, Tobin EJ, Davignon J, Hobbs HH (1990) Common lowdensity lipoprotein receptor mutations in the French Canadian population. J Clin Invest 85: 1014-1023

Leren TP, Solberg K, Rodningen OK, Ose L, Tonstad S, Berg K (1993) Evaluation of running conditions for SSCP analysis: Application of SSCP for detection of point mutations in the LDL receptor gene. PCR Methods Appl 3: 159-162

Mabuchi H, Haba T, Ueda K, Ueda R, Tatami R, Ito S, Kametani T, Koizumi J, Miyamoto S, Ohta M, Takeda R, Takegoshi T, Takeshita $\mathrm{H}$ (1977) Serum lipids and coronary heart disease in heterozygous familial hypercholesterolemia in the Hokuriku district of Japan. Atherosclerosis 28: 417-423

Maruyama T, Miyake Y, Tajima S, Harada-Shiba M, Yamamura T, Tsushima M, Kishino B, Horiguchi Y, Funahashi T, Matsuzawa Y, Yamamoto A (1995) Common mutations in the low-densitylipoprotein-receptor gene causing familial hypercholesterolemia in the Japanese population. Arterioscler Thromb Vasc Biol 15: 17131718

Nakamura Y (1996) Application of DNA markers to clinical genetics. Jpn J Hum Genet 41: 1-14

Russell DW, Esser V, Hobbs HH (1989) Molecular basis of familial hypercholesterolemia. Arteriosclerosis Suppl 9: 1-13

Sudhof TC, Goldstein JL, Brown MS, Russell DW (1985) The LDL receptor gene: A mosaic of exons shared with different proteins. Science 228: 815-822

Williams RR, Hasstedt SJ, Wilson DE, Ash KO, Yanowitz FF, Reiber GE, Kuida H (1986) Evidence that men with familial hypercholesterolemia can avoid early coronary death. JAMA 255 : 219-224

Yanase T (1997) Human genetics: Past, present, and future. Jpn J Hum Genet 2: 265-316 